\title{
Field Effect SnO2 Nano-Thin Film Layer CMOS-Compatible
}

\author{
J.J. Velasco-Vélez ${ }^{1}$, A. Chaiyboun ${ }^{1}$, Ch. Wilbertz ${ }^{2}$, J. Wöllenstein $^{3}$, M. Bauersfeld ${ }^{3}$ and Th. Doll ${ }^{1}$ \\ Johannes-Gutenberg-University Mainz ${ }^{1}$, Micronas $\mathrm{GmbH}^{2}$, Fraunhofer IPM ${ }^{3}$ \\ Institut für Physik, Staudingerweg 7, 55128 Mainz (Germany) velez@uni-mainz.de
}

\section{ABSTRACT:}

The integration of metal oxide gas sensing layers into CMOS electronic still a challenge especially due to the high operating temperatures that do not comply with silicon transistor limits, even more critical, and metal oxide annealing temperatures. External electric fields will allow control over the energy levels of the sensing layer and thus over adsorption sensitivity, consequently the interaction between gas and sensitive layer is modulated. As the absorbed gas on the surface produces a band bending, it changes conduction paths allowing gas detection through resistance measurements. With this configuration, field switch offers fast desorption and thus handling of low temperature response times. Also electric fields may be useful to reduce annealing temperatures. In this paper some aspects as design, measurements and models are studied.

\section{INTRODUCCTION:}

The conventional metal oxide gas sensors consist in a hybrid construction of a sensing element, discrete electronics and package. An integration of these sensors on CMOS technology stills a challenge due the high operation temperatures (up to $700^{\circ} \mathrm{C}$ ), where the electronics can not operate anymore. Some observations of the high electric field leading to sensitivity changes in such devices and the knowledge of early semiconductor surface theory motivated our approach to use these effects for the possible control of a novel ultra thin film gas sensor. These external electric fields open the possibility of an electrical control of the energy levels (Fermi-level) and therefore gas sensitivity modulation on the metal-oxide surface, see Fig.1. Because of this fact, they are enabled to operate with relative low temperature $\left(150^{\circ} \mathrm{C}-200^{\circ} \mathrm{C}\right)$ [1]-[9]. This approach enables the integration into CMOS technology together with the signal processing and information systems and eliminates the need for hybrid system setup.

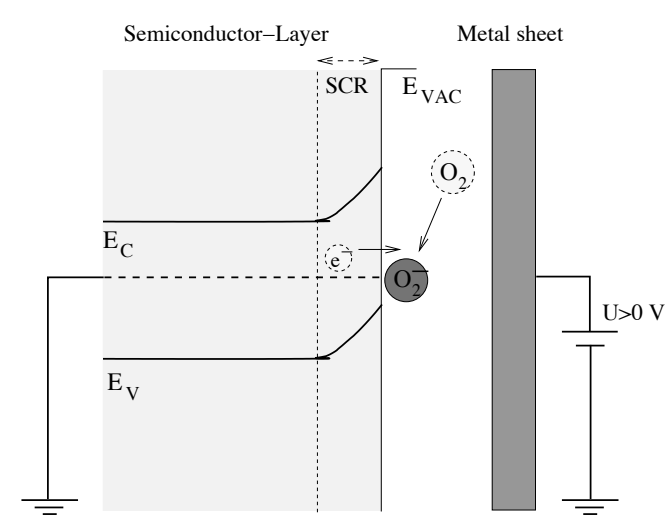

(a)

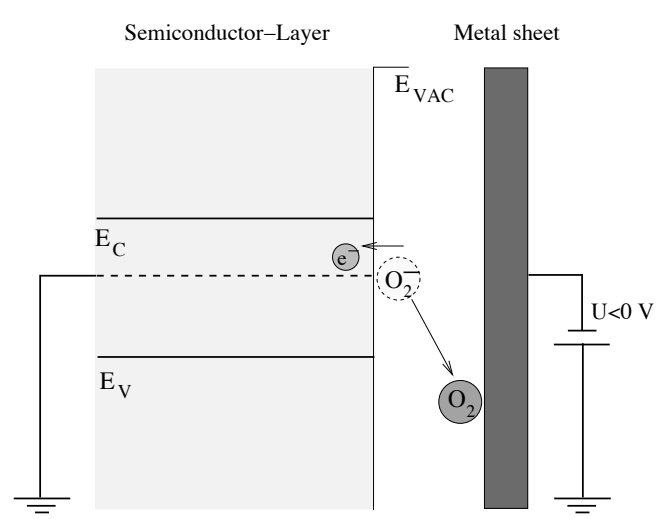

(b)

Fig. 1: (a) Absorption process supported by the application of a positive voltage $U>0 \mathrm{~V}$. (b) desorption process supported by the application of a negative voltage $\mathrm{U}<0 \mathrm{~V}$.

\section{CONCEPT:}

According to previous works [7] nano and micro structures are enabled to reach high electric fields necessary to produce the chemisorption on the surface. In Fig.2.A a concept based on a suspended gate is depicted, external electric fields allow variation on the surface energy levels and consequently producing a change on the gas sensitivity. In order to achieve an effective control of these levels through the sensitivity layer, a reversed insulated-gate field-effect-transistor (IGFET) with the gate 
structure buried in a silicon substrate and the gas sensitive layer as the FET channel deposited on top was developed. In this device Fermi levels position are better controlled as Fig.2.B shows.

(A)

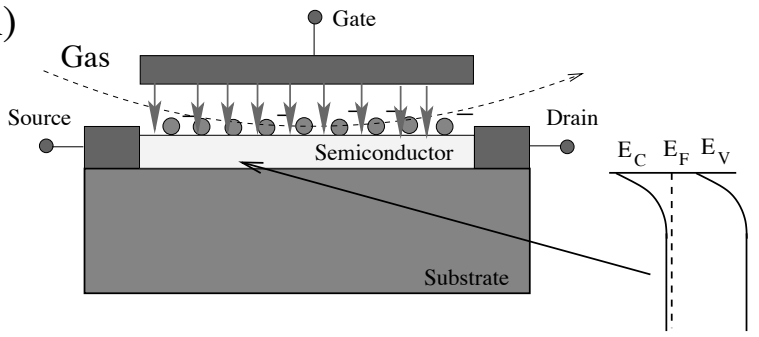

(B)

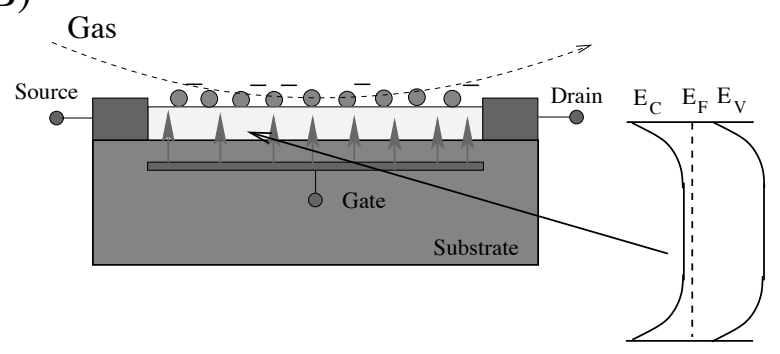

Fig. 2: (A) Suspended gate structure. (B) Buried gate structure.

As the electric field is generated by the gate structure buried in the silicon substrate, stacked sensing layer thickness must be in the range of the Debye length $\left(L_{D}\right)$. It is necessary that the electric field from the buried gate reaches the surface. Only then an effective variation of the Fermi level occurs, producing an excellent control over the adsorption and desorption of all weakly and strongly bound gas molecules according to Wolkenstein adsorption statistics [5]. Sensitivity thickness layer $d$ was sufficiently long compared to $L_{D}$ to enable sensing but short enough to influence unbound molecules [9]. Debye length is given by:

$$
L_{D}=\sqrt{\frac{\varepsilon_{0} \varepsilon_{r} k T}{q^{2} N_{D}}}
$$

Where $\varepsilon_{0}$ is the electric permittivity of vacuum, $\varepsilon_{r}$ is the relative electric permittivity, $K$ is the Boltzmann constant, $T$ represents the temperature, $q$ is the elementary electron charge and $N_{D}$ represents the dopants density.

(A)
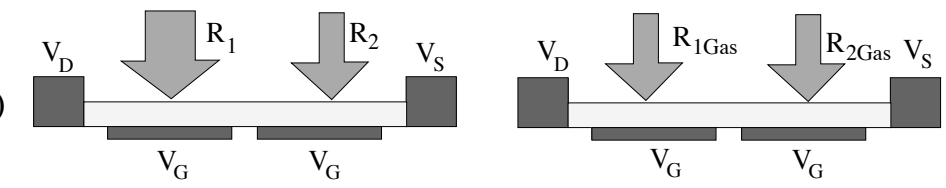

(B)
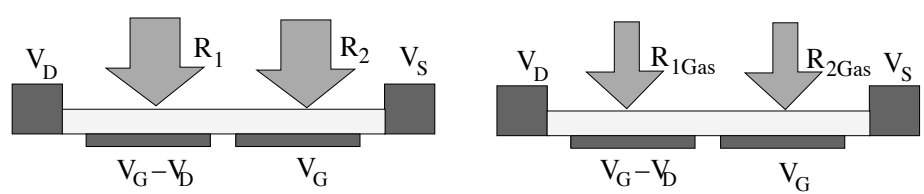

Fig. 3: Ilustration, control of the Fermi energy gradient. (A) IGFET gas sensor with equal gate potential. (B) IGFET gas sensor with different gate potential. Right with gas inlet and left without gas.

The electric field along the channel transistor is not constant under the application of a gate voltage. It occurs because voltages gate-drain and gate-source are different, $V_{G}-V_{D} \neq V_{D}-V_{S}$. Consequently the electric field is inhomogeneous along the longitudinal component resulting in a change in the electron path producing a change in the charge carriers density distribution and therefore a resistance variation. Electric field inhomogeneity also produces an irregular sensitivity behaviour. In order to achieve homogeneity in sensitivity along the channel, multi-gate structure was developed where several gate voltages can be applied. Whit this setting, electrical field along the $\mathrm{SnO} 2$ remains constant and consequently the sensitivity is not varied. The sensitivity $S$ is given by [10]:

$$
S=\frac{\sum_{i=1}^{n} R_{i G a s}}{\sum_{i=1}^{n} R_{i}}
$$

$R_{i G a s}$ is the bulk resistance with gas and $R_{i}$ without gas. 


\section{MEASUREMENTS:}

In order to check sensor operation some measurements were realized under several conditions of gas exposition. In Fig.4 a result is depicted, it is obvious that the sensor shows good response to oxidizing gas $\mathrm{NO}_{2}$ and reducing gas $\mathrm{CO}$. A comparison between the different curves shows that the relative change in the response is higher with negative gate voltage. It means that it is easier to distinguish between the different species, in few words, sensor presents better selectivity.

Measurements with a mix of two gases showed the influence of theses gases at the same time on the sensor response, as shown in Fig.4. For an explanation of this behaviour it is necessary to introduce an extended Wolkenstein's model based on the competition between different species for the absorption centres. This is the object of study in the next section.

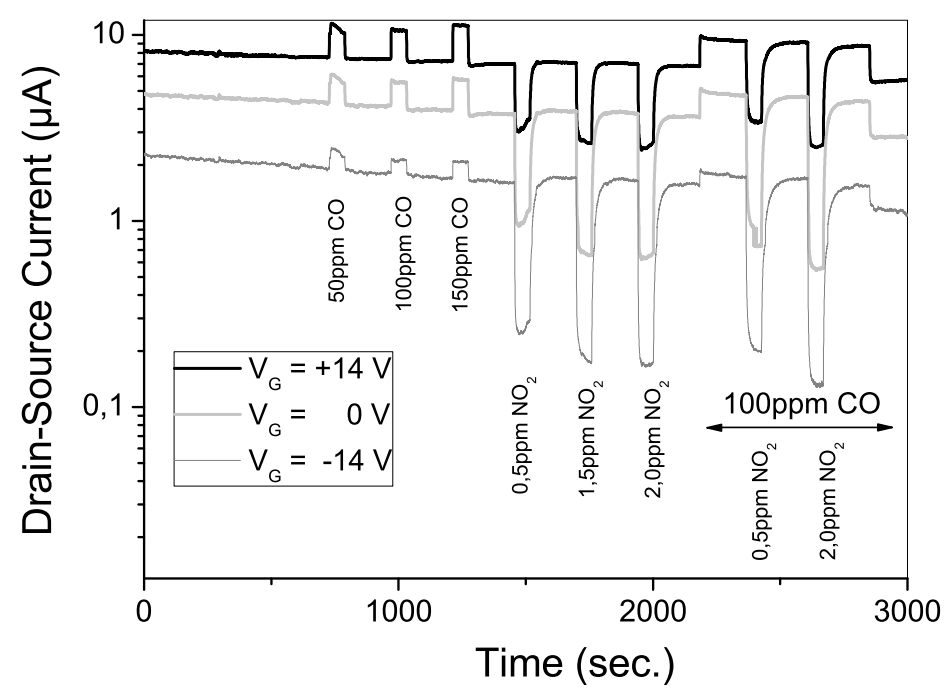

Fig. 4: Right: Drain current measurements under several gate voltage applications. The gas measurements were realized at $\mathrm{T}$ $=200^{\circ} \mathrm{C}$ and $\mathrm{V}_{\mathrm{DS}}=1 \mathrm{~V}$.

Absolute change on the drain current through the application of several gate voltages and $\mathrm{NO}_{2}$ expositions are shown in the Table.1

\begin{tabular}{|c|c|c|c|}
\hline $\mathbf{N O}_{\mathbf{2}}$ & $\boldsymbol{\Delta} \mathbf{I}_{\mathbf{D S}}\left(\mathbf{V}_{\mathbf{G}}=\mathbf{+ 1 4} \mathbf{V}\right)$ & $\Delta \mathbf{I}_{\mathbf{D S}}\left(\mathbf{V}_{\mathbf{G}}=\mathbf{0} \mathbf{V}\right)$ & $\Delta \mathbf{I}_{\mathbf{D S}}\left(\mathbf{V}_{\mathbf{G}}=\mathbf{- 1 4} \mathbf{V}\right)$ \\
\hline $\mathbf{0 . 5} \mathbf{~ p p m}$ & $-3.958 \mu \mathrm{A}$ & $-2.382 \mu \mathrm{A}$ & $-1.359 \mu \mathrm{A}$ \\
\hline $\mathbf{1 . 5} \mathbf{~ p p m}$ & $-4.446 \mu \mathrm{A}$ & $-2.715 \mu \mathrm{A}$ & $-1.530 \mu \mathrm{A}$ \\
\hline $\mathbf{2 . 0} \mathbf{~ p p m}$ & $-4.601 \mu \mathrm{A}$ & $-2.786 \mu \mathrm{A}$ & $-1.565 \mu \mathrm{A}$ \\
\hline
\end{tabular}

Table.1: Absolute current increase through the application of several gate voltages and $\mathrm{NO}_{2}$ concentrations.

Sensor presents better sensitivity to $\mathrm{NO}_{2}$ with positive gate voltage than with the negative gate voltage as the measurements shown.

\section{MODEL}

\subsection{ELECTRICAL CHARACTERIZATION:}

An electrical model is developed using a model based on a Insulate Gate Field Effect Trensistor (IGFET) device with an added surface layer gas reaction. With this configuration, the gas sensitivity is controlled due the application of several gate voltages. Adsortion on surface produces changes in the conductivity of the substrate increasing or decreasing the charge carrier concentration. Because of this fact it is necesary to develope a coupled model with Poisson equation, continuty and Wolkenstenin theory, as shown in previous works [9] 


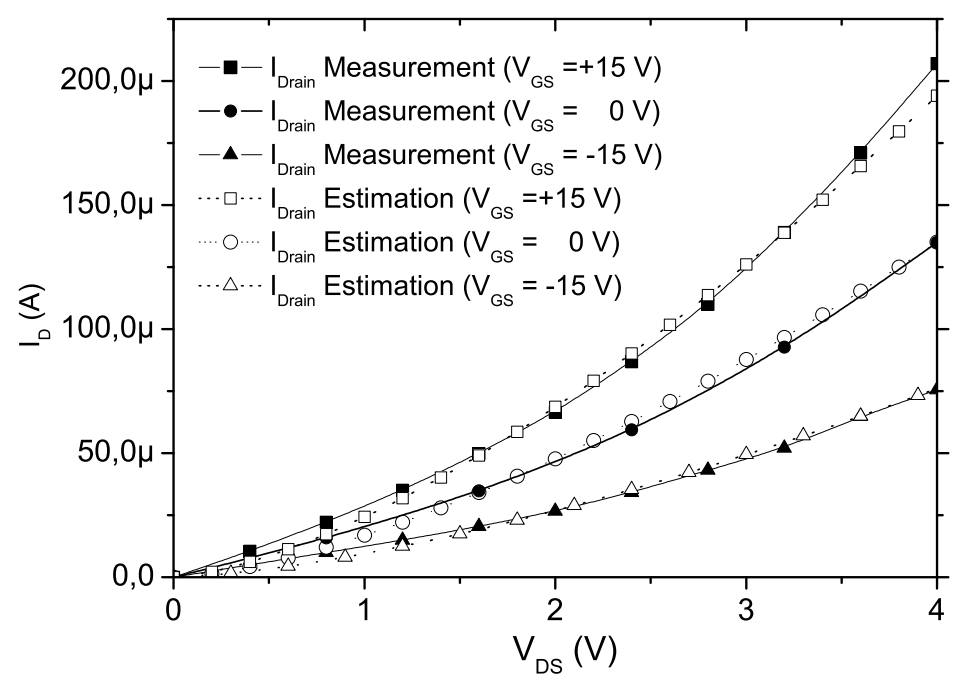

Fig. 5: Drain current comparison between estimation and measurements versus drain-source voltage with different gate voltages. Measurements realized under synthetic air at $40 \%$ relativity humidity at $200^{\circ} \mathrm{C}$.

Fig. 5 shows a comparison between measurements and estimations with good agreement. The nonlineal behaviour in the drain-source current $\left(\mathrm{I}_{\mathrm{DS}}\right)$ depends on the non-homogeneous electric field distribution because the potential difference between drain-gate and source-gate are not the same $\left(\mathrm{V}_{\mathrm{GD}} \neq \mathrm{V}_{\mathrm{GS}}\right)$. It means that the sensor behaves like an IGFET with an added gas sensing layer on the surface.

\subsection{COMPETITIVE ABSORPTION}

With the measurements on the hand it is necessary to introduce a model able to explain the competition between the different species for the absorption centres. Therefore the Wolkenstein model has to be generalized to a number of $\mathrm{i}$ species that do not present reaction between them but compete for the absorption centres on the surface $\left(\mathrm{N}_{\mathrm{ab}}\right)$. With this premise, coverage factor for each specie $\mathrm{i}$ is calculated as:

$$
\Theta=\frac{\beta_{i} \cdot p_{i}}{1+\sum \beta_{i} \cdot p_{i}}
$$

Where $p_{i}$ is the partial pressure for the i specie and $B_{i}$ is the Wolkenstein's isotherm coefficient [11]. The absolute surface charge is equal to the sum of the partial charges then:

$$
N^{-}=\sum N_{i}^{-}=\Theta N_{a b} \quad \bar{i}
$$

with the partial coverage factor $\Theta_{i}^{-}=f_{i}^{-} \Theta_{i}$. Using the most usual chemisorption case where only takes place one electron associated to an energy level $E_{t}$ generally $g_{0}$ times degenerate when it is free and $g_{1}$ is occupied, in this case $g_{0}=2$ and $g_{1}=1$. The strong absorption statistic is given as:

$$
f_{i}^{-}=\frac{1}{1+\frac{g_{0}}{g_{1}} \cdot \exp \left(\frac{E_{t i}-E_{F}}{k T}\right)}
$$

Where $E_{t i}$ is the effective level created by the species i. Consequently the space charge region produced on the surface (interface solid-gas) by chemisorption is given as: 


$$
X_{S C R}=\frac{N^{-}}{N_{D}}
$$

When the sensor is exposed to $\mathrm{NO}_{2}$ the potential produced on the surface through chemisortion is given in Table.2

\begin{tabular}{|c|c|c|c|}
\hline $\mathbf{N O}_{\mathbf{2}}$ & $\mathbf{V}_{\mathbf{S}}\left(\mathbf{V}_{\mathbf{G}}=\mathbf{+ 1 4} \mathbf{~ V}\right)$ & $\mathbf{V}_{\mathbf{S}}\left(\mathbf{V}_{\mathbf{G}}=\mathbf{0} \mathbf{~ V}\right)$ & $\mathbf{V}_{\mathbf{S}}\left(\mathbf{V}_{\mathbf{G}}=\mathbf{- 1 4} \mathbf{~ V}\right)$ \\
\hline $\mathbf{0 . 5} \mathbf{~ p p m}$ & $-0.0326 \mathrm{~V}$ & $-0.0384 \mathrm{~V}$ & $-0.0416 \mathrm{~V}$ \\
\hline $\mathbf{1 . 5} \mathbf{~ p p m}$ & $-0.0384 \mathrm{~V}$ & $-0.0549 \mathrm{~V}$ & $-0.0634 \mathrm{~V}$ \\
\hline $\mathbf{2 . 0} \mathbf{p p m}$ & $-0.0416 \mathrm{~V}$ & $-0.0634 \mathrm{~V}$ & $-0.0898 \mathrm{~V}$ \\
\hline
\end{tabular}

Table.2: Surface potential produces by strong chemisorption.

and its correspondent strongly chemisorbed surface particles density is given in Table.3

\begin{tabular}{|c|c|c|c|}
\hline $\mathbf{N O}_{\mathbf{2}}$ & $\mathbf{N}_{\mathbf{S}}\left(\mathbf{V}_{\mathbf{G}}=\mathbf{+ 1 4} \mathbf{V}\right)$ & $\mathbf{N}_{\mathbf{S}}\left(\mathbf{V}_{\mathbf{G}}=\mathbf{0} \mathbf{V}\right)$ & $\mathbf{V}_{\mathbf{S}}\left(\mathbf{V}_{\mathbf{G}}=\mathbf{- 1 4} \mathbf{~ V}\right)$ \\
\hline $\mathbf{0 . 5} \mathbf{~ p p m}$ & $1.8908 \times 10^{16} \mathrm{~m}^{-2}$ & $2.0522 \times 10^{16} \mathrm{~m}^{-2}$ & $2.1359 \times 10^{16} \mathrm{~m}^{-2}$ \\
\hline $\mathbf{1 . 5} \mathbf{~ p p m}$ & $2.0522 \times 10^{16} \mathrm{~m}^{-2}$ & $2.4538 \times 10^{16} \mathrm{~m}^{-2}$ & $2.6160 \times 10^{16} \mathrm{~m}^{-2}$ \\
\hline $\mathbf{2 . 0} \mathbf{~ p p m}$ & $2.1359 \times 10^{16} \mathrm{~m}^{-2}$ & $2.6369 \times 10^{16} \mathrm{~m}^{-2}$ & $3.1382 \times 10^{16} \mathrm{~m}^{-2}$ \\
\hline
\end{tabular}

Table.3: Surface density of strongly chemisorbed particles.

Table. 3 shows how the number of strongly chemisorbed particles is increased with an increase in the $\mathrm{NO}_{2}$ density, meaning that the length of the space charge region on the surface is increased, as Table.2 shows implicit. On the other hand, when the gate voltage is positive it produces a decrease on the density of strongly chemisorbed particles but with a negative gate voltage the density is increased $\left(\Theta_{\mathrm{NO} 2}\right.$ $=0.6535 ; \mathrm{f}_{\mathrm{NO} 2}=0.8665 ; \mathrm{N}_{\mathrm{NO} 2}^{-}=2.6650 \times 10^{16} \mathrm{~m}^{-2}$ for $\mathrm{V}_{\mathrm{G}}=0 \mathrm{~V}$ and $2.0 \mathrm{ppm}$ of $\mathrm{NO}_{2}$ ), in accordance with theory. Consequently the sensor sensitivity is higher. More results related to other aspects will be provided on the conference.

\section{DISSCUSION:}

The influence of an external electric field application on gas sensitivity was studied through an electrical characterization of the different processes involved. It was developed by means of a model based on an IGFET with a multi-gate structure.

The most important implication is the gate voltage influence on the selectivity. To achieve this control gate dielectric and sensing layer thickness must be in the Debye length $\left(L_{D}\right)$ range. Then the small dimensions offer the opportunity of a better absorption-desorption modulation on the surface at the solid-gas interface. Therefore it is possible to reduce the operation temperature and to integrate the sensor into CMOS circuitry. In summary, these results are of general nature and can be considered helpful in understanding chemisorption processes under the application of external electric fields in very small devices (nanometer lengths) like in new thin film metal-oxides, nanowires and polymer electronics.

\section{ACKNOWLEDGEMENTS:}

This work was supported by the German Ministry of Education and Research (BMBF) under Contract $16 S V 2170$.

\section{REFERENCES}

[1] B. Weisz, "Effects of Electronic Charge Transfer between Adsorbate and Solid on Chemisorption and Catalysis", J. Chem. Phys. 21, 9, pp. 1531-1538, 1953.

[2] F. F. Wolkenstein, W. B. Sandomirski, Dokl. Acad. Nauk. SSSR, 118, pp. 980-982, 1958. 
[3] H. Geistlinger , "Chemisorption effects on the thin-film conductivity", Surface Science 277, pp. 429441, North Holland 1992.

[4] L. I. Popova, S. K. Andreev, V. K. Guoerguiev, N. D. Stoyanov, "Voltage dependence of gas-sensing behaviour of SnO2- gate FETs", Sens. Act. B, 18/19, pp. 543-545, 1994.

[5] M. Hausner, J. Zacheja, J. Binder, "Multi-Electrode Substrate foSelectivity Enhancement in Air Monitoring", Sens. Act. B, 172, 180, 1997.

[6] W. Hellmich, G. Muller, V. Bosch, C. Braunmuhl, Th. Doll , I. Eisele , "Field-Effect-Induced Gas Sensitivity Changes in Metal Oxides", Sens. Actuators B 43, pp. 132-139, 1997.

[7] M. Boegner, A. Fuchs, K. Scharnagl, R. Winter, Th. Doll, I. Eisele, "Electrical field impact on the gas adsorptivity of thin metal oxide films", Appl. Phys. Let., 73, 17, 1998.

[8] Th. Doll, Advanced Gas Sensing: The Electroadsorptive Effect and Related Techniques, Boston, Kluwer, 2003

[9] J. J. Velasco-Velez, A. Chaiyboun, C. Wilbertz, J. Wöllenstein, M. Bauersfeld, T. Doll, "CMOSCompatible Nanoscale Gas-Sensor Based on Field-Effecf', Physica Status Solidi (a), Accepted 20/11/2008.

[10] J. Wöllenstein, Zur Selektivitätssteigerung von Halbleitergassensoren, Shaker Verlag, Aachen, 2003.

[11] J. Santos, P. Serrini, B. O'Beirn, L.Manes, "A thin film $\mathrm{SnO}_{2}$ gas sensor selective to ultra-low $\mathrm{NO}_{2}$ concentrations in air", Sens. And Act. B 43, pp. 156-160, 1997. 